\title{
Barrierefreiheit in Kindergärten und Schulen
}

\author{
Inklusion von blinden und sehbehinderten Kindern und \\ Jugendlichen erfordert spezielle Maßnahmen.
}

Barrierefreier Lebensraum ist ein zentraler Faktor, auch wenn es um Inklusion von Kindern und Jugendlichen geht. Bei der Gestaltung von Kindergärten, Schulen und Spielplätzen ist demnach zu beachten, dass die Umsetzung von Barrierefreiheit in vollem Umfang erfolgt. Um Lösungen zu schaffen, die in der Praxis gut funktionieren, ist die Beratung mit der Zielgruppe unbedingt erforderlich, da betroffene Personen ein wertvolles Spektrum in die Beurteilung von Problemen, Lösungsansätzen und Alternativen einbringen können, das als nicht-betroffene Personen, auch Fachexperten, nur schwer erlangt werden kann.

\section{Zugang zu Bildung}

Inklusion und gleichberechtigte Teilhabe im Bildungsbereich sind von elementarer Bedeutung für Gleichberechtigung im gesellschaftlichen Leben. Der barrierefreie Zugang zu Bildung ist essentiell für Personen mit Behinderung, um später wirtschaftliche Unabhängigkeit erlangen zu können. Die Forderung nach pädagogischen, personellen und materiellen Rahmenbedingungen, die Kindern mit Sehbehinderung, Blindheit und Mehrfachbehinderung eine gelungene Teilhabe an und eine gute, ihren individuellen Bedürfnissen entsprechende, Schulbildung ermöglicht, ergibt sich also ganz natürlich.

\section{Frühförderung}

Eine gute Frühförderung wird hierbei vorausgesetzt. Durch sie wird dem blinden oder sehbehinderten Kind die Welt nahe gebracht. Mittels Spielanregungen und Fördermaterialien können hier Impulse gesetzt werden, die Anregun- gen für alle Sinnesbereiche bieten. Die Entwicklung des Kindes wird dadurch unterstützt, dass es Gegenstände und deren Funktionsweisen mit sämtlichen Sinnen untersucht und so Handlungsabläufe kennenlernt. Die Förderung des Sehens ist ein wesentlicher Bereich der Frühförderung, der nur unter barrierefreien Bedingungen stressfrei und zielführend verfolgt werden kann.

\section{Das Bewusstsein wächst ...}

Erfreulicherweise ist derzeit in Österreich ein großes Bestreben erkennbar, im Sinne der Vorgaben der UN Konvention über die Rechte von Menschen mit Behinderungen sowie des Bundes-Behindertengleichstellungsgesetzes, Maßnahmen zu setzen. Das betrifft auch den Bereich barrierefreien Bauens. Es ist ein großer Erfolg, dass das Bewusstsein für die Relevanz barrierefreier Gestaltung immer größer wird. Im Zuge dessen wird auch die Forderung nach einem barrierefreien Umfeld immer seltener als außergewöhnlich angesehen, sondern als berechtigte Grundlage aufgefasst.

\section{Barrierefreies Bauen und Gestalten}

Erst das Zusammenspiel der verschiedenen Aspekte von Barrierefreiheit (Stufenlosigkeit, Bewegungsraum, Orientierungsmöglichkeiten, Sicherheitsvorkehrungen, etc.) ermöglichen ein wirklich inklusives bauliches Umfeld und damit gesellschaftliche Partizipation für alle Menschen. Daher ist es unbedingt erforderlich, im Kontext barrierefreien Bauens und Gestaltens, ein wesentlich größeres Augenmerk auf Maßnahmen im Sinne blinder und sehbehinderter Menschen zu legen, als es derzeit der Fall ist.
Wenn es um Inklusion geht ... Barrierefreier Lebensraum ist ein wesentlicher Faktor, wenn es um Inklusion geht. Einerseits ist das Maß an Barrierefreiheit, ein Ausdruck dessen, inwieweit die Gesellschaft den Inklusionsgedanken verinnerlicht hat, andererseits beeinflusst die Art, wie Lebensraum gestaltet ist, die Menschen, die sich darin aufhalten und bewegen, maßgeblich. Je weniger Barrieren es gibt, desto weniger werden Menschen mit verschiedenen Beeinträchtigungen von ihrem Umfeld behindert. Die Folge wäre ein freieres und selbständigeres Agieren in allen Lebensbereichen, größere gesellschaftliche Präsenz und eine selbstverständlichere gleichberechtigte Teilhabe am gesellschaftlichen Leben. Das hätte wiederum brächte mit sich, dass Barrierefreiheit als Qualitätskriterium anerkannt und nicht mehr hinterfragt, sondern von allen Menschen aktiv eingefordert würde.

\section{Zukunftsmusik?}

Ist diese Vorstellung utopisch? Mit Sicherheit nicht. Haben wir es hier mit Zukunftsmusik zu tun? Vielleicht. Sollten wir uns dafür einsetzen, dass sie so schnell wie möglich Wirklichkeit wird? Ja, auf jeden Fall, besonders auch dort, wo Kinder erhöhten Schutz und Unterstützung benötigen, in Kindergärten und Schulen.

Paediatr. Paedolog. Austria 2016 · 51:223 DOI 10.1007/s00608-016-0414-4 Online publiziert: 6. Oktober 2016 (c) Springer-Verlag Wien 2016

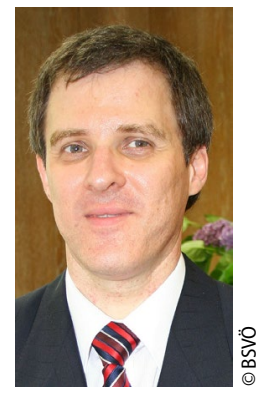

Dr. Markus Wolf, Präsident des Blinden- und Sehbehindertenverbandes Österreich 